\title{
Sex differences in morphometric aspects of the peripheral nerves and related diseases
}

\author{
Hiroshi Moriyama $^{\mathrm{a}, *}$, Shogo Hayashi ${ }^{\mathrm{b}}$, Yuriko Inoue ${ }^{\mathrm{a}}$, Masahiro Itoh $^{\mathrm{b}}$ and Naruhito Otsuka ${ }^{\mathrm{a}}$ \\ ${ }^{a}$ Department of Anatomy, Showa University School of Medicine, Tokyo, Japan \\ ${ }^{\mathrm{b}}$ Department of Anatomy, Tokyo Medical University, Tokyo, Japan
}

\begin{abstract}
.
BACKGROUND: The elucidation of the relationship between the morphology of the peripheral nerves and the diseases would be valuable in developing new medical treatments on the assumption that characteristics of the peripheral nerves in females are different from those in males.

METHODS: We used 13 kinds of the peripheral nerve. The materials were obtained from 10 Japanese female and male cadavers. We performed a morphometric analysis of nerve fibers. We estimated the total number of myelinated axons, and calculated the average transverse area and average circularity ratio of myelinated axons in the peripheral nerves.

RESULTS: There was no statistically significant difference in the total number, average transverse area, or average circularity ratio of myelinated axons between the female and male specimens except for the total number of myelinated axons in the vestibular nerve and the average circularity ratio of myelinated axons in the vagus nerve.

CONCLUSIONS: The lower number of myelinated axons in the female vestibular nerve may be one of the reasons why vestibular disorders have a female preponderance. Moreover, the higher average circularity ratio of myelinated axons in the male vagus nerve may be one reason why vagus nerve activity to modulate pain has a male preponderance.
\end{abstract}

Keywords: Peripheral nerve, sex difference, morphometry, axon, human, neurological disorder

\section{Introduction}

There are many reports of sex difference, little sex difference, and no sex difference in the incidence of peripheral nerve diseases and peripheral nerve damage after surgical procedures. However, there are few sex difference reports concerning the relationship between the morphology of the peripheral nerves and the related diseases. Acquiring these reports would be valuable in not only understanding gender-specific medicine but also developing new medical treatments on the assumption that characteristics of the peripheral nerves in females are different

*Address for correspondence: Hiroshi Moriyama, DDS, PhD, Department of Anatomy, Showa University School of Medicine, 5-8, Hatanodai 1, Shinagawa-ku, Tokyo 142-8555, Japan. Tel.: +81 33784 8107; Fax: +81 35498 7800; E-mail: veloce@med.showau.ac.jp. from those in males. Therefore, we morphometrically analyzed peripheral nerves and clarified the relationship between the morphology of the peripheral nerves and the diseases in this study.

\section{Materials and methods}

\subsection{Materials}

Human oculomotor nerves, ophthalmic nerves, inferior alveolar nerves, abducent nerves, facial nerves, vestibular nerves, cochlear nerves, vagus nerves, and recurrent laryngeal nerves in the cranial nerve group; femoral nerves and tibial nerves in the spinal nerve group; and greater splanchnic nerves and lesser splanchnic nerves in the autonomic nerve group were resected with epifascicular epineurium. The materials were obtained from 10 Japanese female and 10 Japanese male cadavers aged 54-90 years 
(average: 75.2). All the cadavers were donated with the individual's consent. We proceeded to perform this research in accordance with the law concerning autopsy and preservation of corpses, and also donation for medical and dental education. In no case was there a history of peripheral nerve disorders such as neuroparalysis or schwannoma, or of treatment with toxic agents or irradiation therapy. The causes of death did not directly or indirectly influence the nervous system, so the peripheral nerves were considered to be normal. We used right side specimens of righthanded persons to avoid any interaction between the effects of sex and side. Moreover, female and male groups were each composed of 10 cadavers of the same age $(54,61,68,70,75,78,83,85,88,90$ years) to avoid any interaction between the effects of sex and age.

\subsection{Methods}

The preparation of sections involved fixation, washing, dehydration, embedding, and sectioning, as described in our previous report (Moriyama et al., 2007a). All the cadavers were fixed with a $10 \%$ solution of formalin ( $3.7 \%$ formaldehyde) within 24 hours of postmortem. After resecting the peripheral nerves, a $10 \%$ solution of formalin ( $3.7 \%$ formaldehyde) was used for immersion for at least a week. The solution was changed once in the first 30 to $60 \mathrm{~min}$, and again later if desired. The formalin-fixed materials were then transferred, without washing, to the secondary fixative to be held at room temperature for two weeks. If the solution became turbid or precipitated it was changed. After this, the fixation was continued at $37^{\circ} \mathrm{C}$ for an additional week. The volume of fixative used was at least ten times the volume of the specimens. After washing, dehydration, and celloidin embedding, we cut sections $15-\mu \mathrm{m}$ thick and stained them with modified luxol fast blue-periodic acid Schiff-hematoxylin triple stain.

\subsection{Morphometry}

We observed the microscopic section at low power (Fig. 1), and covered the entire area of the distributed myelinated axons in the peripheral nerve by moving the eyepiece grid vertically and horizontally as described in our previous report (Moriyama et al., 2007b). We confirmed that we could distinguish myelinated structures from vessels in the tissue with a computer or grouped unmyelinated axons with the naked eye in each grid. We counted the myeli-

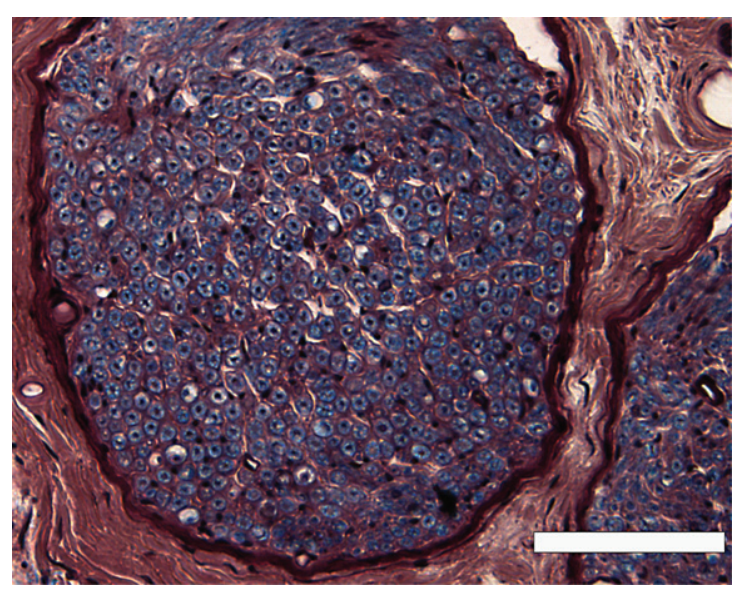

Fig. 1. A low-power view of the inferior alveolar nerve from a 78-year-old man, modified luxol fast blue-periodic acid Schiffhematoxylin triple stain. Scale bar $=100 \mu \mathrm{m}$.

nated axons and measured the transverse area and perimeter of the myelinated axons in a square eyepiece grid at high power (Fig. 2). To avoid duplicate counts, we counted and measured all axons on the side of the grid that did not come into contact with the other grids. In the case of grids adjacent to other grids, we counted and measured only the axons on the lower right side of the grid, not those on the upper left side. We used a microscope in transmitted light mode (BX50, Olympus, Tokyo, Japan) equipped with a high-resolution digital camera (ColorView12, Soft Imaging System, Münster, Germany), a motorized XYZ stage (Märzhäuser, Wetzlar-Steindorf, Germany), a stage controller (Märzhäuser, WetzlarSteindorf, Germany), and a computer (Precision 530, Dell, Austin, TX, USA) with analyzing system software (analySIS 3.0, Soft Imaging System, Münster, Germany) to store data on-line, do calculations, and perform statistical analyses. Circularity ratios were calculated as follows: circularity ratio $=4 \pi \mathrm{A} / \mathrm{L}^{2}$ $\left(\mathrm{A}=\right.$ area in $\mathrm{mm}^{2} ; \mathrm{L}=$ perimeter in $\mathrm{mm}$ ).

If a circle is regular, the ratio has a maximum value of 1.0, and if it is irregular, the value is less than 1. This indicates how near or far each irregularity is from a regular round figure, allowing quantitative comparison of round figures.

\subsection{Statistical analyses}

All statistical analyses were performed using JMP statistical software version 9.0.3 (SAS Institute Inc. Cary, NC, USA) on a Macintosh personal computer. 


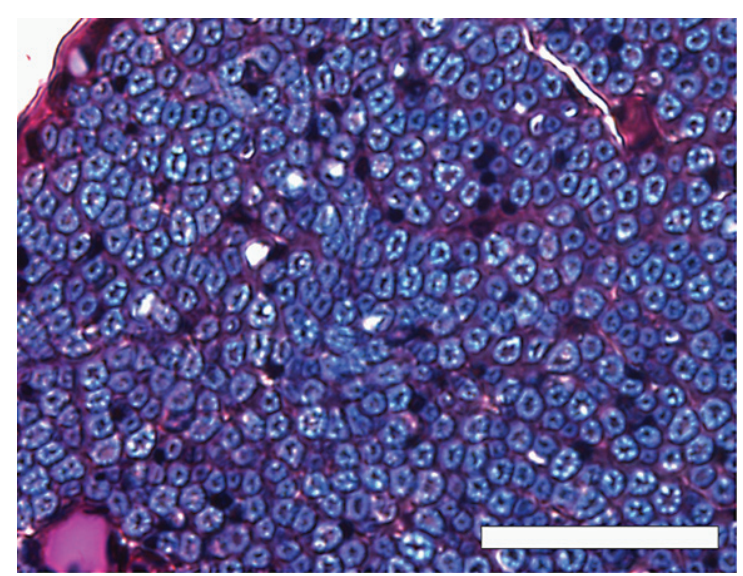

Fig. 2. A high-power view of the inferior alveolar nerve from a 68-year-old man, modified luxol fast blue-periodic acid Schiffhematoxylin triple stain. Axons were stained dark purple or black, and surrounded by a myelin sheath stained indigo blue. Scale bar $=50 \mu \mathrm{m}$.

Researchers have studied shrinkage of embedding materials, and found that celloidin and plastination embedding exhibit less shrinkage (around 10\%) than paraffin and other embeddings (Eckel et al., 1993). Therefore, although we measured every myelinated axon, we calculated the average transverse area and perimeter of myelinated axons after excluding data far from the median (15\%) due to shrinkage. Morphological differences between female and male specimens were analyzed by applying a parametric unpaired $t$ test (where data were normally distributed with equal variance) to the total number or number/unit area of myelinated axons, the average transverse area and circularity ratio. A $p$ value of $<0.05$ was considered to indicate a statistically significant difference.

\section{Results}

\subsection{Number of myelinated axons}

We estimated the total number or number per unit area of myelinated axons in the peripheral nerves (Table 1). The myelinated nerve fibers appeared as a indigo blue myelin sheath surrounding a dark purple or black axon (Fig. 1). According to the data, there was no statistically significant difference in the total number of myelinated axons, between the female and male specimens, in the peripheral nerves, except for the vestibular nerve $(P<0.05$; Table 1$)$.

\subsection{Average transverse area of myelinated axons}

The average transverse area of myelinated axons in the peripheral nerves was calculated (Table 1). According to the data, there was no statistically significant difference in the average transverse area of myelinated axons, between the female and male specimens, in all calculated peripheral nerves $(P<0.05$; Table 1).

\subsection{Circularity ratio of myelinated axons}

The average circularity ratio of myelinated axons in the peripheral nerves was calculated (Table 1) and there was no statistically significant difference in the average circularity ratio of myelinated axons, between the female and male specimens, in the peripheral nerves, except for the vagus nerve $(P<0.05$; Table 1$)$.

\section{Discussion}

Researchers have reported that a decrease in the number and size of myelinated axons influences the occurrence of peripheral nerve palsy or neuropathy (Korinthenberg et al., 1997; Fukuda et al., 2000; Lin \& Soong, 2002; Griffn \& Höke, 2005), and a lower circularity ratio of myelinated axons has been partly implicated in the degeneration of nerve fibers (Moriyama et al., 1995). A smaller number, average transverse area, and lower average circularity ratio of myelinated axons would help explain the sex difference in the incidence of peripheral nerve palsy and neuropathy.

Cabrejas et al. reported from epidemiological data on oculomotor nerve palsy that there were $59.1 \%$ males, with no statistically significant difference between females and males $(p=0.574)$ (Cabrejas et al., 2009). Moreover, Ohguro et al. reported finding differences in oculomotor nerve palsy with causative disease incidence according to sex, but they reported finding no significant difference in oculomotor nerve palsy with unknown cause incidence according to sex (Ohguro et al., 1986). According to the data in this study, there was no statistically significant difference in the total number, average transverse area, or average circularity ratio of myelinated axons between the female and male specimens of the oculomotor nerve $(P<0.05$; Table 1$)$. The findings in this paper may partly explain why there is no significant sex difference in the incidence of oculomotor nerve palsy. 
Table 1

Comparison of myelinated axons in the peripheral nerves between females and males

\begin{tabular}{|c|c|c|c|c|c|}
\hline Nerve & Sex & $\begin{array}{c}\text { Number } \\
\text { of Specimens }\end{array}$ & $\begin{array}{c}\text { Total Number } \\
\text { or Number/Unit area }\end{array}$ & $\begin{array}{c}\text { Average Transverse } \\
\text { Area }\left(\mu \mathrm{m}^{2}\right)\end{array}$ & $\begin{array}{c}\text { Average } \\
\text { Circularity Ratio } \\
\end{array}$ \\
\hline \multirow[t]{4}{*}{ Oculomotor } & $\mathrm{F}$ & 10 & $18,305 \pm 3,669$ & $6.14 \pm 1.09$ & $0.83 \pm 0.04$ \\
\hline & M & 10 & $19,640 \pm 4,996$ & $6.39 \pm 1.18$ & $0.85 \pm 0.03$ \\
\hline & $p$ value & & $P=0.52$ & $P=0.73$ & $P=0.20$ \\
\hline & $95 \% \mathrm{CI}$ & & $-2,784$ to 5,452 & -0.82 to 1.32 & -0.01 to 0.06 \\
\hline \multirow[t]{4}{*}{ Ophthalmic } & F & 10 & $32 \pm 9\left(16 \times 16 \mu \mathrm{m}^{2}\right)$ & $4.16 \pm 1.64$ & $0.84 \pm 0.04$ \\
\hline & M & 10 & $37 \pm 9\left(16 \times 16 \mu \mathrm{m}^{2}\right)$ & $5.18 \pm 1.10$ & $0.86 \pm 0.05$ \\
\hline & $p$ value & & $P=0.24$ & $P=0.14$ & $P=0.34$ \\
\hline & $95 \% \mathrm{CI}$ & & -4 to 13 & -0.29 to 2.33 & -0.02 to 0.06 \\
\hline \multirow[t]{4}{*}{ Inferior Alveolar } & $\mathrm{F}$ & 10 & $21,910 \pm 8,128$ & $33.60 \pm 8.29$ & $0.87 \pm 0.03$ \\
\hline & M & 10 & $23,633 \pm 7,046$ & $30.83 \pm 9.23$ & $0.86 \pm 0.05$ \\
\hline & $p$ value & & $P=0.62$ & $P=0.88$ & $P=0.94$ \\
\hline & $95 \% \mathrm{CI}$ & & $-5,423$ to 8,869 & -11.01 to 5.47 & -0.05 to 0.03 \\
\hline \multirow[t]{4}{*}{ Abducent } & F & 10 & $1,861 \pm 360$ & $4.13 \pm 0.58$ & $0.79 \pm 0.08$ \\
\hline & M & 10 & $2,110 \pm 506$ & $3.98 \pm 0.47$ & $0.77 \pm 0.05$ \\
\hline & $p$ value & & $P=0.27$ & $P=0.38$ & $P=0.65$ \\
\hline & $95 \% \mathrm{CI}$ & & -164 to 660 & -0.65 to 0.35 & -0.09 to 0.04 \\
\hline \multirow[t]{4}{*}{ Facial } & $\mathrm{F}$ & 10 & $6,157 \pm 579$ & $6.64 \pm 0.83$ & $0.80 \pm 0.07$ \\
\hline & M & 10 & $6,567 \pm 738$ & $6.25 \pm 0.69$ & $0.79 \pm 0.06$ \\
\hline & $p$ value & & $P=0.16$ & $P=0.36$ & $P=0.70$ \\
\hline & $95 \% \mathrm{CI}$ & & -213 to 1,033 & -1.10 to 0.33 & -0.07 to 0.05 \\
\hline \multirow[t]{4}{*}{ Vestibular } & $\mathrm{F}$ & 10 & $18,488 \pm 2,725^{*}$ & $3.64 \pm 0.56$ & $0.88 \pm 0.04$ \\
\hline & M & 10 & $22,321 \pm 3,448^{*}$ & $3.60 \pm 1.01$ & $0.86 \pm 0.08$ \\
\hline & $p$ value & & $P=0.02$ & $P=0.91$ & $P=0.76$ \\
\hline & $95 \% \mathrm{CI}$ & & 914 to 6,753 & -0.81 to 0.73 & -0.08 to 0.04 \\
\hline \multirow[t]{4}{*}{ Cochlear } & $\mathrm{F}$ & 10 & $23,183 \pm 4,192$ & $1.80 \pm 0.30$ & $0.86 \pm 0.02$ \\
\hline & M & 10 & $25,937 \pm 5,234$ & $1.83 \pm 0.24$ & $0.85 \pm 0.02$ \\
\hline & $p$ value & & $P=0.24$ & $P=0.94$ & $P=0.32$ \\
\hline & $95 \% \mathrm{CI}$ & & $-1,701$ to 7,209 & -0.23 to 0.29 & -0.03 to 0.01 \\
\hline \multirow[t]{4}{*}{ Vagus } & $\mathrm{F}$ & 10 & $1,456 \pm 315$ & $1.10 \pm 0.27$ & $0.79 \pm 0.06^{*}$ \\
\hline & M & 10 & $1,320 \pm 209$ & $1.27 \pm 0.32$ & $0.85 \pm 0.04^{*}$ \\
\hline & $p$ value & & $P=0.47$ & $P=0.31$ & $P=0.04$ \\
\hline & $95 \% \mathrm{CI}$ & & -388 to 115 & -0.11 to 0.45 & 0.01 to 0.11 \\
\hline \multirow[t]{4}{*}{ Recurrent Laryngeal } & $\mathrm{F}$ & 10 & $14 \pm 2 / 33 \times 33 \mu \mathrm{m}^{2}$ & $10.31 \pm 2.16$ & $0.87 \pm 0.04$ \\
\hline & M & 10 & $15 \pm 3 / 33 \times 33 \mu \mathrm{m}^{2}$ & $10.68 \pm 1.98$ & $0.90 \pm 0.04$ \\
\hline & $p$ value & & $P=0.47$ & $P=0.79$ & $P=0.22$ \\
\hline & $95 \% \mathrm{CI}$ & & -1 to 3 & -1.58 to 2.32 & -0.01 to 0.06 \\
\hline \multirow[t]{4}{*}{ Femoral } & $\mathrm{F}$ & 10 & $28 \pm 12 / 33 \times 33 \mu \mathrm{m}^{2}$ & $5.62 \pm 1.56$ & $0.90 \pm 0.07$ \\
\hline & M & 10 & $32 \pm 11 / 33 \times 33 \mu \mathrm{m}^{2}$ & $6.19 \pm 1.68$ & $0.91 \pm 0.05$ \\
\hline & $p$ value & & $P=0.47$ & $P=0.38$ & $P=0.79$ \\
\hline & $95 \% \mathrm{CI}$ & & -7 to 15 & -0.96 to 2.09 & -0.05 to 0.07 \\
\hline \multirow[t]{4}{*}{ Tibial } & $\mathrm{F}$ & 10 & $1,389 \pm 468$ & $2.64 \pm 0.42$ & $0.93 \pm 0.04$ \\
\hline & M & 10 & $1,550 \pm 573$ & $2.95 \pm 0.40$ & $0.91 \pm 0.07$ \\
\hline & $p$ value & & $P=0.62$ & $P=0.12$ & $P=0.54$ \\
\hline & $95 \% \mathrm{CI}$ & & -330 to 653 & -0.08 to 0.70 & -0.07 to 0.03 \\
\hline \multirow[t]{4}{*}{ Greater Splanchnic } & F & 10 & $9 \pm 2 / 16 \times 16 \mu \mathrm{m}^{2}$ & $5.58 \pm 1.38$ & $0.86 \pm 0.07$ \\
\hline & M & 10 & $8 \pm 2 / 16 \times 16 \mu \mathrm{m}^{2}$ & $5.81 \pm 0.74$ & $0.88 \pm 0.07$ \\
\hline & $p$ value & & $P=0.54$ & $P=0.12$ & $P=0.70$ \\
\hline & $95 \% \mathrm{CI}$ & & -3 to 1 & -0.81 to 1.28 & -0.05 to 0.08 \\
\hline \multirow{4}{*}{ Lesser Splanchnic } & $\mathrm{F}$ & 10 & $12 \pm 6 / 16 \times 16 \mu \mathrm{m}^{2}$ & $1.63 \pm 0.96$ & $0.85 \pm 0.10$ \\
\hline & M & 10 & $12 \pm 4 / 16 \times 16 \mu \mathrm{m}^{2}$ & $1.66 \pm 0.66$ & $0.83 \pm 0.12$ \\
\hline & $p$ value & & $P=0.88$ & $P=0.71$ & $P=0.57$ \\
\hline & $95 \% \mathrm{CI}$ & & -4 to 5 & -0.75 to 0.80 & -0.12 to 0.09 \\
\hline
\end{tabular}

*Indicates a significant difference $(p<0.05)$. Each value is the mean \pm SD. CI, confidence interval.

Researchers have reported that the incidence rate of trigeminal neuralgia was slightly higher for females than for males. For example, the female-to-male ratio was $1.74: 1$ in the Katusic et al. study (Katusic et al., 1990) and $3: 2$ in another study by Ashkenazi and Levin (Ashkenazi \& Levin, 2004). It has been proposed that the symptoms of trigeminal neuralgia are caused by demyelination of the nerve 
leading to ephaptic transmission of impulses. Surgical specimens have demonstrated this demyelination and close apposition of demyelinated axons in the trigeminal root of patients with trigeminal neuralgia (Love \& Coakham, 2001). Results from experimental studies suggest that demyelinated axons are prone to ectopic impulses, which may transfer from light touch to pain fibers in close proximity (ephaptic conduction) (Love \& Coakham, 2001). Current theories regarding the cause of this demyelination center on vascular compression of the nerve root by aberrant or tortuous vessels. Accepting current theories, neurovascular (or microvascular) decompression, when a pad is placed between a vessel and the nerve, has been found empirically to be an effective treatment for trigeminal neuralgia in cases resistant to medical therapy, and as many as $90 \%$ of cases have been relieved (Apfelbaum, 1988; Jannetta, 1990). Barker et al. reported that 706 patients (around 60\%) were female of 1185 patients who underwent microvascular decompression, and female sex was a risk factor for recurrence after microvascular decompression (hazard ratio 1.3; $P=0.06$ ) (Barker et al., 1996). According to the data here, there was no statistically significant difference in the number per unit area, average transverse area, or average circularity ratio of myelinated axons between the female and male specimens of the ophthalmic nerve $(P<0.05$; Table 1). Therefore, a vascular abnormality in the female trigeminal neuralgia may be one reason why trigeminal neuralgia has a female preponderance, but morphology in the characteristic nerve does not appear to explain the sex difference in the incidence of trigeminal neuralgia.

Inferior alveolar nerve damage can occur after an inferior alveolar nerve block (Lambrianidis \& Molyvdas, 1987; Pogrel \& Thamby, 2000) or following oral and maxillofacial surgical procedures (Panula et al., 2001; Kraut \& Chahal, 2002; TeerijokiOksa et al., 2003). With regard to the incidence of inferior alveolar nerve damage after these procedures, Haas and Lennon reported no significant sex difference (ratio of affected females to males, $72: 68$ ) (Haas \& Lennon, 1995). Kipp et al. also reported that the incidence was $7 \%$ in both sexes, indicating no significant sex difference (Kipp et al., 1980), while Harn and Durham reported that there was little sex difference in postinjection lingual nerve injuries (ratio of affected females to males, 24 : 17) (Harn \& Durham, 1990). Meanwhile, sexual dimorphism that results in the incidence being almost twice as high in females than in males has been reported (Pogrel \&
Thamby, 2000; Queral-Godoy et al., 2005). Pogrel and Thamby found the difference in referral rates for male and female patients difficult to explain (Pogrel $\&$ Thamby, 2000). They mentioned that there have been studies suggesting that nerves respond differently to injury in female animals than in male animals (Wagner et al., 1995). Coyle et al. reported that female rats were more prone to developing tactile allodynia than male rats after partial sciatic nerve ligation (Coyle et al., 1995). These reports may partially explain the indicated sex difference in the incidence of inferior alveolar nerve damage (Coyle et al., 1995; Wagner et al., 1995). According to this study's data, there was no statistically significant difference in the total number, average transverse area, or average circularity ratio of myelinated axons between the female and male inferior alveolar nerve specimens $(P<0.05$; Table 1). Heasman and Beynon reported a difference between the total number of myelinated axons in the human inferior alveolar nerve of dentate and edentulous groups as significant $(P<0.001)$ and suggested axonal atrophy in the main nerve trunk following tooth loss (Heasman \& Beynon, 1987). As each cadaver in this study had 7 teeth (central incisor, lateral incisor, canine, first premolar, second premolar, first molar, and second molar) on the side of the mandible that we used, we considered that this result was not affected by the dentulous condition. Therefore, the results of this study suggest a nonsignificant sex difference in the incidence of inferior alveolar nerve damage, supported by the morphometric analysis. These findings may partly explain why there is no significant sex difference in the incidence of inferior alveolar nerve damage.

Patel et al. reported 69 male (50\%) and 68 female $(50 \%)$ cases of abducent nerve palsy or paresis (Patel et al., 2004). According to this study, the average transverse area in the female abducent nerve was larger than that in the male abducent nerve, but there was no statistically significant difference in the total number, average transverse area, or average circularity ratio of myelinated axons between the female and male specimens of the abducent nerve $(P<0.05$; Table 1). Our findings may partly explain why there is no significant sex difference in the incidence of abducent nerve palsy or paresis.

Campbell and Brundage reported that the incidence rate of Bell's palsy was slightly higher for females than for males (rate ratio $=1.16$ ) $($ Campbell \& Brundage, 2002). Meanwhile, Monini et al. reported that males were slightly more affected (53.7\%) than females (Monini et al., 2010). However, 
many researchers have reported finding no significant difference in Bell's palsy incidence according to sex, as Tiemstra and Khatkhate recently reported (Tiemstra \& Khatkhate, 2007). In this study, there was no statistically significant difference in the total number, average transverse area, or average circularity ratio of myelinated axons, between the female and male facial nerve specimens $(P<0.05$; Table 1$)$. These findings may partly explain why there is no significant sex difference in the incidence of Bell's palsy.

The incidence of vestibular dysfunction has a female preponderance in a textbook description (Hullar et al., 2005). There was a marked female preponderance among individuals with vestibular vertigo (one year prevalence ratio female to male of $2.7: 1.0$ ) (Lempert \& Neuhauser, 2009). Neuhauser et al. also reported that prevalence and incidence rates of vestibular vertigo were consistently higher in females than in males, for example, the lifetime prevalence ratio of female to male was $10.3: 4.3$, and the population incidence ratio (one year) female to male was 1.9: 0.8 (Neuhauser et al., 2005). This female preponderance tended to increase with age (Yin et al., 2009). Yin et al. reported that adults (18-65y) had a ratio of affected females to males of $59.1: 40.9$, but elderly adults $(>65 \mathrm{y})$ had a ratio of $60.6: 39.4$ (Yin et al., 2009). With regard to Menière's disease, a female preponderance can be assumed based on the data from Rochester (61\% women) (Wladislavosky-Waserman et al., 1984) and is confirmed by the latest data from Finland (Havia et al., 2005). The results here showed that total number was 3,833 (mean value) higher in the male vestibular nerve than the female vestibular nerve. Our data also indicated a significant sex difference $(P=0.02$; Table 1$)$, but there was no statistically significant difference in the average transverse area and average circularity ratio of myelinated axons between the female and male vestibular nerve specimens $(P<0.05$; Table 1$)$. The lower number of myelinated axons in the female vestibular nerve may be one of the reasons why vestibular disorders have a female preponderance, but the findings here on the average transverse area and average circularity ratio of myelinated axons did not appear to explain the sex difference in the incidence of these diseases.

There are some reports regarding the incidence of cochlear dysfunction with sex difference. The incidence of tinnitus has a female preponderance in a textbook description (Davis, 1987). Meanwhile, Graham (Graham, 1965) and the National Study of Hearing (Coles, 1984) reported that the incidence rate of tinnitus was higher for females than for males until the mid-fifties, but that after the mid-fifties, tinnitus was higher for males than for females. Møller et al. reported that of the 72 patients who underwent microvascular decompression of the intracranial portion of the auditory nerve, $54.8 \%$ experienced total relief from tinnitus or marked improvement (Møller et al., 1993). This report indicated that vascular compression of the auditory nerve was a factor in tinnitus. Therefore, vascular abnormalities in tinnitus patients may be one reason why tinnitus shows a sex difference, but morphology in the characteristic nerve does not appear to explain the sex difference in the incidence of tinnitus. Next, with regard to the incidence of hearing acuity, Kacker reported that there was no significant sex difference (Kacker, 1997). Meanwhile, Hinchcliffe and Jones reported that the hearing acuity in males was better than that in females (Hinchcliffe \& Jones 1968). Researchers also reported that the differences in hearing levels between females and males depended on the frequencies or race (Bunch \& Raiford, 1931; Berger et al., 1977). Moreover, Star et al. reported that there were four females and six males among 10 patients with auditory nerve disease (Starr et al., 1996). The main lesion in auditory nerve disease is thought to be demyelination or degeneration of cochlear nerve fibers. Finally, Nakashima examined the nationwide epidemiological study of sudden deafness in 1993, and reported that there was no significant sex difference (Nakashima, 2001). In this study, there was no statistically significant difference in the total number, average transverse area, or average circularity ratio of myelinated axons between the female and male cochlear nerve specimens $(P<0.05$; Table 1). The findings here may partly explain why there is no significant sex difference in the incidence of cochlear dysfunction.

Araújo et al. assessed vagal activity using heart rate response to a short bicycle exercise test during maximal inspiratory apnea. This study aimed to evaluate the role of sex and physical activity patterns on vagal activity. As a result, no sex effect could be identified (Araújo et al., 1989). With regard to vagoglossopharyngeal neuralgia, researchers reported that there was no preponderance regarding sex (Bruyn, 1983; Kandan et al., 2010). We gave a supplementary explanation for the term "vagoglossopharyngeal neuralgia". As researchers took the central or peripheral overlap between the glossopharyngeal nerve and vagus nerve into consideration, they grouped glossopharyngeal and vagal neuralgia together, and used the more useful vagoglossopharyngeal neuralgia in 
clinical practice (Crue \& Todd, 1968; Rushton et al., 1981). Meanwhile, Khasar et al. reported that under normal conditions, responses to noxious stimuli were modulated by vagus nerve activity in males, but not in females (Khasar et al., 2001). Our results showed that the average circularity ratio was 0.06 (mean value) higher in the male vagus nerve than the female vagus nerve. Our data also indicated a significant sex difference $(P=0.04$; Table 1$)$. However, there was no statistically significant difference in the total number and average transverse area of myelinated axons between the female and male vagus nerve specimens $(P<0.05$; Table 1$)$. The higher average circularity ratio of myelinated axons in the male vagus nerve may be one reason why vagus nerve activity to modulate pain has a male preponderance. Our findings regarding the total number and average transverse area of myelinated axons may partly explain why there is no significant sex difference in the incidence of vagoglossopharyngeal neuralgia.

With respect to sex, males with recurrent laryngeal nerve paralysis were more frequent than females in some reports (Parnell \& Brandenburg, 1970; Titche, 1976; Yamada et al., 1983), whereas in some other reports (Work, 1941; Huppler et al., 1955; Hirose et al., 1981), there were more females than males with that condition. However, overall the above data indicated that there were 1,526 females with recurrent laryngeal nerve paralysis (48.5\%) and 1,618 males $(51.5 \%)$ (Yamada et al., 1983). Therefore, there was no significant difference in the number of patients between the two sexes. In this study, there was no statistically significant difference in the total number, average transverse area, or average circularity ratio of myelinated axons between the female and male recurrent laryngeal nerve specimens $(P<0.05$; Table 1). These findings may partly explain why there is no significant sex difference in the incidence of recurrent laryngeal nerve paralysis.

Shinoda analyzed data on adult motor neuropathy in past reports, and mentioned that the vulnerability of male motor neurons was higher than that of female motor neurons (Shinoda, 1998). With regard to amyotrophic lateral sclerosis (ALS), a progressive disorder of the motor neurons, the incidence of ALS was slightly higher for males than for females (male/female rate was $2.0 \%$ or less) in reports including 100 cases or more. For example, Bonduelle et al. (Bonduelle et al., 1970), Boman and Meurman (Boman and Meurman, 1967), and Kondo (Kondo, 1975) reported that the male/female rate ratio was $1.2,1.3,1.5$, respectively. According to our data, there was no statistically significant difference in the total number, average transverse area, or average circularity ratio of myelinated axons between the female and male femoral and tibial nerve specimens $(P<0.05$; Table 1). These findings may partly explain why there is little significant sex difference in the incidence of motor neuropathy. Therefore, sex difference in the incidence of motor neuropathy is considered to be caused not only by the morphology of the motor neurons, but also because of sex hormones and other factors.

Shinoda analyzed data on adult autonomic neuropathy in past reports and mentioned that the vulnerability of male autonomic neurons was higher than that of females (Shinoda, 1998). Hogarth and coworkers' study demonstrated that females have lower central sympathetic nerve activity to the periphery, the mechanism of which involves a greater baroreceptor reflex inhibitory effect on this activity in females than in males (Hogarth et al., 2007). These findings could have implications regarding the lower number of cardiovascular events observed in females than in males. Muneta et al. reported that the activation of the sex center regulating gonadotropin secretion may be a causative factor in the baroreflex impairment in females (Muneta et al., 1994). They also mentioned that changes in blood pressure in females are more sensitive to mental stress, but less so to isometric stress, than those of males. These findings suggest that ovarian dysfunction is another important factor influencing the baroreflex function in addition to aging and blood pressure, and that the baroreflex impairment in females characterizes the sex difference in the pathophysiology of essential hypertension. Hinojosa-Laborde et al. reported that clear evidence exists for differences in the regulation of the sympatho-adrenal nervous system between males and females (Hinojosa-Laborde et al., 1999). At each level of neural control examined in their review, females were able to limit the activation or enhance the inhibition of the sympathetic nervous system more effectively than males during at least part of the oestral/menstrual cycle. These observations suggest that the ability of females to more tightly control the sympathetic nervous system and, subsequently, arterial pressure may serve as a mechanism whereby sex hormones protect females against hypertension. Here, there was no statistically significant difference in the total number, average transverse area, or average circularity ratio of myelinated axons, between the female and male specimens, of the greater splanchnic and lesser splanchnic nerve 
$(P<0.05$; Table 1$)$. These findings do not explain why there is a significant sex difference in the incidence of autonomic dysfunction. Therefore, the morphology of autonomic neurons may not be the cause of sex differences in the incidence of autonomic dysfunction, and other factors such as sex hormones may be the cause.

\section{Conclusions}

We morphometrically analyzed nine kinds of the nerve in the cranial nerve group, two kinds of the nerve in the spinal nerve group, and two kinds of the nerve in the autonomic nerve group in this study. There was no statistically significant difference in the total number, average transverse area, or average circularity ratio of myelinated axons between the female and male specimens of 11 kinds of the peripheral nerve except for the vestibular and vagus nerve $(P<0.05$; Table 1). Our data indicated a significant sex difference in the total number $(P=0.02$; Table 1$)$, but there was no statistically significant difference in the average transverse area and average circularity ratio of myelinated axons between the female and male vestibular nerve specimens $(P<0.05$; Table 1$)$. The lower number of myelinated axons in the female vestibular nerve may be one of the reasons why vestibular disorders have a female preponderance. Moreover, our data indicated a significant sex difference in the average circularity ratio $(P=0.04$; Table 1), but there was no statistically significant difference in the total number and average transverse area of myelinated axons between the female and male vagus nerve specimens $(P<0.05$; Table 1$)$. The higher average circularity ratio of myelinated axons in the male vagus nerve may be one reason why vagus nerve activity to modulate pain has a male preponderance. Our findings regarding the total number and average transverse area of myelinated axons may partly explain why there is no significant sex difference in the incidence of vagoglossopharyngeal neuralgia.

\section{Acknowledgments}

We thank Ms. Ikuko Moriyama for assistance in preparing this manuscript. This work was supported by a Grant-in-aid for Scientific Research B14370007 from the Ministry of Education, Culture, Sports, Science and Technology of Japan.

\section{Financial support and disclosure}

We certify that there are no affiliations or involvement (either competitive or amiable) with any organization or entity with a direct financial interest in the subject matter or materials discussed in the manuscript (e.g., employment, consultancies, stock ownership, honoraria, expert testimony, etc.). This work was supported by a Grant-in-aid for Scientific Research B14370007 from the Ministry of Education, Culture, Sports, Science and Technology of Japan. The corresponding author had full access to all the data in the study and had final responsibility for the decision to submit for publication.

\section{Conflict of interest}

We declare that we have no conflicts of interest.

\section{References}

Apfelbaum, R. (1988). Surgical management of disorders of the lower cranial nerves. In H. Schmideck \& W. Sweet (Eds.), Operative neurosurgical techniques (2nd ed.) (pp. 1097-1109). New York, NY: Grune \& Stratton.

Araújo, C. G., Nobrega, A. C., \& Castro, C. L. (1989). Vagal activity: Effect of age, sex and physical activity pattern. Braz J Med Biol Res, 22, 909-211.

Ashkenazi, A., \& Levin, M. (2004). Three common neuralgias. How to manage trigeminal, occipital, and postherpetic pain. Postgrad Med, 116, 16-32.

Barker, F. G. II, Jannetta, P. J., Bissonette, D. J., Larkins, M. V., \& Jho, H. D. (1996). The long-term outcome of microvascular decompression for trigeminal neuralgia. N Engl J Med, 334, 1077-1083.

Berger, E. H., Royster, L. H., \& Thomas, W. G. (1977). Hearing levels of nonindustrial exposed subjects. J Occup Med, 19, 664-670.

Boman, K., \& Meurman, T. (1967). Prognosis of amyotrophic lateral sclerosis. Acta Neurol Scand, 43, 489-498.

Bonduelle, M., Bouygues, P., Lormeau, G., \& Keller, J. (1970). Clinical and developmental study of 125 cases of amyotrophic lateral sclerosis: Nosographic limitations and morbid associations. Presse Med, 78, 827-832.

Bruyn, G. W. (1983). Glossopharyngeal neuralgia. Cephalalgia, 3, 143-157.

Bunch, C. C., \& Raiford, T. S. (1931). Race and sex variations in auditory acuity. Arch Otolaryngol, 13, 423-434.

Cabrejas, L., Hurtado-Ceña, F. J., \& Tejedor, J. (2009) Predictive factors of surgical outcome in oculomotor nerve palsy. J AAPOS, 13, 481-484.

Campbell, K. E., \& Brundage, J. F. (2002). Effects of climate, latitude, and season on the incidence of Bell's palsy in the US armed forces, October 1997 to September 1999. Am J Epidemiol, 156, 32-39.

Coles, R. R. A. (1984). Epidemiology of tinnitus: Demographic and clinical features. J Laryngol Otology, (Suppl 9), 195-202. 
Coyle, D. E., Sehlhorst, C. S., \& Mascari, C. (1995). Female rats are more susceptible to the development of neuropathic pain using the partial sciatic nerve ligation (PSNL) model. Neurosci Lett, 186, 135-138.

Crue, B. L., \& Todd, E. M. (1968). Vagal neuralgia. In: P. J. Vinken \& G. W. Bruyn (Eds.), Headaches and cranial neuralgias (pp. 362-367). Amsterdam, Netherlands: North-Holland Pub. Co.

Davis, B. (1987). The incidence of tinnitus. In: R. Slater, M. Terry, \& B. Davis (Eds.) Tinnitus: A guide for sufferers and professionals (88-98). Kent, UK: Croom Helm Ltd.

Eckel, H. E., Sittel, C., Walger, M., Sprinzl, G., \& Koebke, J. (1993). Plastination: A new approach to morphological research and instruction with excised larynges. Ann Otol Rhinol Laryngol, 102, 660-665.

Fukuda, M., Morimoto, T., Suzuki, Y., Kida, K., \& Ohnishi, A. (2000). Congenital neuropathy with the absence of large myelinated fibers. Pediatr Neurol, 23, 349-351.

Graham, J. T. (1965). Relation of tinnitus to age. Acta Otolaryngol, 59, 24-26.

Griffn, J. W., \& Höke, A. (2005). The control of axonal caliber. In: P. J. Dyck \& P. K. Thomas, (Eds.), Peripheral Neuropathy vol. 1 (4th ed.) (pp. 433-446). Philadelphia: Elsevier Saunders.

Haas, D. A., \& Lennon, D. (1995). A 21 year retrospective study of reports of paresthesia following local anesthetic administration. J Can Dent Assoc, 61, 319-320, 323-326, 329-330.

Harn, S. D., \& Durham T. M. (1990). Incidence of lingual nerve trauma and postinjection complications in conventional mandibular block anesthesia. J Am Dent Assoc, 121, 519-523.

Havia, M., Kentala, E., \& Pyykkö, I. (2005). Prevalence of Menière's disease in general population of Southern Finland. Otolaryngol Head Neck Surg, 133, 762-768.

Heasman, P. A., \& Beynon, A. D. G. (1987). Myelinated axon counts of human inferior alveolar nerves. J Anat, 151, 51-56.

Hinchcliffe, R., \& Jones, W. I. (1968). Hearing levels of a suburban Jamaican population. Int $J$ Audiol, 7, 239-258.

Hinojosa-Laborde, C., Chapa, I., Lange, D., \& Haywood, J. R. (1999). Gender differences in sympathetic nervous system regulation. Clin Exp Pharmacol Physiol, 26, 122-126.

Hirose, H., Sawashima, M., \& Yoshioka, H. (1981). Clinical observations on 750 cases of laryngeal palsy. Ann Bull RILP, 15, 173-180.

Hogarth, A. J., Mackintosh, A. F., \& Mary, D. A. (2007). Genderrelated differences in the sympathetic vasoconstrictor drive of normal subjects. Clin Sci (Lond), 112, 353-361.

Hullar, T. E., Minor, L. B., \& Zee, D. S. (2005). Evaluation of the patient with dizziness. In C. W. Cummings (Ed.), Cummings otolaryngology head \& neck surgery vol. 4 (4th ed.) (pp. 31603198). Philadelphia: Elsevier Mosb.

Huppler, E. G., Schmidt, H. W., \& Devine, D. (1955). Causes of vocal-cord paralysis. Proc Staff Meet Mayo Clin, 30, 518-521.

Jannetta, P. J. (1990). Cranial rhizopathies. In J. R. Youmans (Ed.), Neurological surgery (3rd Ed.) (pp. 4169-4182). Philadelphia: WB Saunders.

Kacker, S. K. (1997). Hearing impairment in the aged. Indian J Med Res, 106, 333-339.

Kandan, S. R., Khan, S., Jeyaretna, D. S., Lhatoo, S., Patel, N. K., \& Coakham, H. B. (2010). Neuralgia of the glossopharyngeal and vagal nerves: Long-term outcome following surgical treatment and literature review. Br J Neurosurg, 24, 441-446.

Katusic, S., Beard, C. M., Bergstralh, E., \& Kurland, L. T. (1990). Incidence and clinical features of trigeminal neuralgia, Rochester, Minnesota, 1945-1984. Ann Neurol, 27, 89-95.
Khasar, S. G., Isenberg, W. M., Miao, F. J., Gear, R. W., Green, P. G., \& Levine, J. D. (2001). Gender and gonadal hormone effects on vagal modulation of tonic nociception. J Pain, 2, 91-100.

Kipp, D. P., Goldstein, B. H., \& Weiss, W. W. Jr. (1980). Dysesthesia after mandibular third molar surgery: A retrospective study and analysis of 1,377 surgical procedures. J Am Dent Assoc, 100, 185-192.

Kondo, K. (1975). Clinical variability of motor neuron disease. Neurological Medicine, 2, 11-16.

Korinthenberg, R., Sauer, M., Ketelsen, U. P., Hanemann, C. O., Stoll, G., Graf, M.,... \& Zerres, K. (1997). Congenital axonal neuropathy caused by deletions in the spinal muscular atrophy region. Ann Neurol, 42, 364-368.

Kraut, R. A., \& Chahal, O. (2002). Management of patients with trigeminal nerve injuries after mandibular implant placement. J Am Dent Assoc, 133, 1351-1354.

Lambrianidis, T., \& Molyvdas, J. (1987). Paresthesia of the inferior alveolar nerve caused by periodontalendodontic pathosis. Oral Surg Oral Med Oral Pathol, 63, 90-92.

Lempert, T., \& Neuhauser, H. (2009). Epidemiology of vertigo, migraine and vestibular migraine. J Neurol, 256, 333-338.

Lin, K. P., \& Soong, B. W. (2002). Peripheral neuropathy of Machado-Joseph disease in Taiwan: A morphometric and genetic study. Eur Neurol, 48, 210-217.

Love, S., \& Coakham, H. B. (2001). Trigeminal neuralgia: Pathology and pathogenesis [published correction appears in Brain (2002). 125, 687]. Brain, 124, 2347-2360.

Møller, M. B., Møller, A. R., Jannetta, P. J., \& Jho, H. D. (1993). Vascular decompression surgery for severe tinnitus: Selection criteria and results. Laryngoscope, 103, 421-427.

Monini, S., Lazzarino, A. I., Iacolucci, C., Buffoni, A., \& Barbara, M. (2010). Epidemiology of Bell's palsy in an Italian Health District: Incidence and case-control study. Acta Otorhinolaryngol Ital, 30, 198-204.

Moriyama, H., Shimada, K., \& Goto, N. (1995). Morphometric analysis of neurons in ganglia: Geniculate, submandibular, cervical spinal and superior cervical. Okajimas Folia Anat Jpn, 72, 185-190

Moriyama, H., Amano, K., Itoh, M., Shimada, K., \& Otsuka, N. (2007). Morphometric aspects of peripheral nerves in adults and the elderly. J Peripher Nerv Syst, 12, 205-209.

Moriyama, H., Shimada, K., Itoh, M., Takahashi, T., \& Otsuka, N. (2007). Morphometric analysis of the inferior alveolar nerve fails to demonstrate sexual dimorphism. J Oral Maxillofac Surg, 65, 1555-1561.

Muneta, S., Murakami, E., \& Hiwada, K. (1994). Gender difference in blood pressure regulation in essential hypertension. Hypertens Res, 17, 71-78.

Nakashima, T. (2001). Sudden deafness. In: Y. Nomura, A., Komatsuzaki, \& I., Honjyo (Eds.), Clinical textbooks of the ear, nose and throat regions 21, No. 5. (pp. 259-269). Tokyo: Nakayama Shoten Co. Ltd.

Neuhauser, H. K., von Brevern, M., Radtke, A., Lezius, F., Feldmann, M., Ziese, T. \& Lempert, T. (2005). Epidemiology of vestibular vertigo: A neurotologic survey of the general population. Neurology, 65, 898-904.

Ohguro, H., Takeda, M., \& Nakagawa, T. (1986) Acquired oculomotor nerve palsy: A review of 74 cases. Neuro-ophthalmol Jpn, 3, 263-266. 
Panula, K., Finne, K., \& Oikarinen, K. (2001). Incidence of complications and problems related to orthognathic surgery: A review of 655 patients. J Oral Maxillofac Surg, 59, 1128-1136.

Parnell, F. W., \& Brandenburg, J. H. (1970). Vocal cord paralysis: A review of 100 cases. Laryngoscope, 80, 1036-1045.

Patel, S. V., Mutyala, S., Leske, D. A., Hodge, D. O., \& Holmes, J. M. (2004). Incidence, associations, and evaluation of sixth nerve palsy using a population-based method. Ophthalmology, $111,369-375$.

Pogrel, M. A., \& Thamby, S. (2000). Permanent nerve involvement resulting from inferior alveolar nerve blocks. JAm Dent Assoc, 131, 901-907.

Queral-Godoy, E., Valmaseda-Castellon, E., Berini-Aytes, L., \& Gay-Escoda, C. (2005). Incidence and evolution of inferior alveolar nerve lesions following lower third molar extraction. Oral Surg Oral Med Oral Pathol Oral Radiol Endod, 99, 259264.

Rushton, J. G., Stevens, J. C., \& Miller, R. H. (1981). Glossopharyngeal (vagoglossopharyngeal) neuralgia: A study of 217 cases. Arch Neurol, 38, 201-205.

Shinoda, K. (1998). Sex difference in adult neuropathy. Sex Difference and Similarity, 4, 34-44.

Starr, A., Picton, T. W., Sininger, Y., Hood, L. J., \& Berlin, C. I. (1996). Auditory neuropathy. Brain, 119, 741-753.
Teerijoki-Oksa T., Jääskeläinen, S., Forssell, K., Virtanen, A, Forssell, H. (2003). An evaluation of clinical and electrophysiology tests in nerve injury diagnosis after mandibular sagittal split osteotomy. Int J Oral Maxillofac Surg, 32, 15-23.

Tiemstra, J. D., \& Khatkhate, N. (2007). Bell's palsy: Diagnosis and management. Am Fam Physician, 76, 997-1002.

Titche, L. L. (1976). Causes of recurrent laryngeal nerve paralysis. Arch Otolaryngol, 102, 259-261.

Wagner, R., DeLeo, J. A., Coombs, D. W., \& Myers, R. R. (1995). Gender differences in autotomy following sciatic cryoneurolysis in the rat. Physiol Behav, 58, 37-41.

Wladislavosky-Waserman, P., Facer, G. W., Mokri, B., \& Kurland, L. T. (1984). Menière's disease: A 30-year epidemiologic and clinical study in Rochester, Mn, 1951-1980. Laryngoscope, 94, 1098-1102.

Work, W. P. (1941). Paralysis and paresis of the vocal cords: A statistical review. Arch Otolaryngol, 34, 267-280.

Yamada, M., Hirano, M., \& Ohkubo, H. (1983). Recurrent laryngeal nerve paralysis: A 10 -year review of 564 patients. Auris Nasus Larynx, 10(Suppl), S1-S15.

Yin, M., Ishikawa, K., Wong, W. H., \& Shibata, Y. (2009). A clinical epidemiological study in 2169 patients with vertigo. Auris Nasus Larynx, 36, 30-35. 